\title{
Nrf2 activation drive macrophages polarization and cancer cell epithelial- mesenchymal transition during interaction
}

\author{
Rui Feng, Yuji Morine*, Tetsuya Ikemoto, Satoru Imura, Shuichi Iwahashi, Yu Saito and Mitsuo Shimada
}

\begin{abstract}
Background: The M2 phenotype of tumor-associated macrophages (TAM) inhibits the anti-tumor inflammation, increases angiogenesis and promotes tumor progression. The transcription factor Nuclear Factor (erythroid-derived 2)-Like 2 (Nrf2) not only modulates the angiogenesis but also plays the anti-inflammatory role through inhibiting pro-inflammatory cytokines expression; however, the role of Nrf2 in the cancer cell and macrophages interaction is not clear.
\end{abstract}

Methods: Hepatocellular carcinoma cells (Hep G2 and Huh 7) and pancreatic cancer cells (SUIT2 and Panc-1) were co-cultured with monocytes cells (THP-1) or peripheral blood monocytes derived macrophages, then the phenotype changes of macrophages and epithelial-mesenchymal transition of cancer cells were detected. Also, the role of Nrf2 in cancer cells and macrophages interaction were investigated.

Results: In this study, we found that cancer cells could induce an M2-like macrophage characterized by up-regulation of CD163 and Arg1, and down-regulation of IL-1b and IL-6 through Nrf2 activation. Also, Nrf2 activation of macrophages promoted VEGF expression. The Nrf2 activation of macrophages correlated with the reactive oxygen species induced by cancer cells derived lactate. Cancer cells educated macrophages could activate Nrf2 of the cancer cells, in turn, to increase cancer cells epithelial-mesenchymal transition (EMT) through paracrine VEGF. These findings suggested that Nrf2 played the important role in the cancer cells and macrophages interaction.

Conclusions: Macrophage Nrf2 activation by cancer cell-derived lactate skews macrophages polarization towards an M2like phenotype and educated macrophages activate Nrf2 of the cancer cells to promote EMT of cancer cells. This study provides a new understanding of the role of Nrf2 in the cancer cell and TAM interaction and suggests a potential therapeutic target.

Keywords: Nuclear factor (erythroid-derived 2)-like 2, Tumor-associated macrophage, Hepatocellular carcinoma, Pancreatic cancer, Epithelial-mesenchymal transition

\section{Background}

The tumor microenvironment, mainly consisted of extracellular matrix, fibroblasts, vascular endothelial cells and immunocytes, plays an important role in tumor structure and biology, contributing tumor cells survival, proliferation and migration [1]. Macrophages are attracted and activated by tumor microenvironment derived cytokines, differentiating into tumor-associated macrophages (TAMs)

\footnotetext{
* Correspondence: ymorine@tokushima-u.ac.jp

Department of Surgery, Institute of Biomedical Sciences, Tokushima

University of Graduate School, 3-18-15 Kuramoto-cho, Tokushima 770-8503, Japan
}

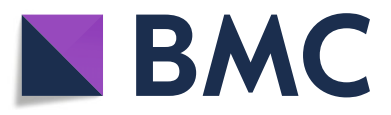

(c) The Author(s). 2018 Open Access This article is distributed under the terms of the Creative Commons Attribution 4.0 International License (http://creativecommons.org/licenses/by/4.0/), which permits unrestricted use, distribution, and reproduction in any medium, provided you give appropriate credit to the original author(s) and the source, provide a link to the Creative Commons license, and indicate if changes were made. The Creative Commons Public Domain Dedication waiver (http://creativecommons.org/publicdomain/zero/1.0/) applies to the data made available in this article, unless otherwise stated. [2]. It has indicated that TAMs were multifunctional and heterogeneous [3]. The one phenotype of TAMs shows the pro-inflammatory characteristic is identified as classical or M1 macrophages, which secret IL-1 and IL-6; the other phenotype owns anti-inflammatory capability referred to alternative or M2 macrophages, which mainly express Arg1 and CD163 [4]. The current studies have shown that higher amount of M2 TAMs was associated with worse clinical prognosis [5-7]. A higher density of M2 TAMs appears to correlate with tumor cell proliferation, metastasis, and intra-tumoral microvascular density $[8,9]$.

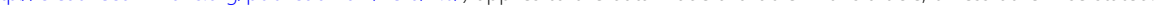


Nuclear factor (erythroid-derived 2)-like 2 (Nrf2), which is essential for keeping the balance of oxidative stress, has been known to improve cell survival under threaten of xenobiotic toxicants through initiating anti-oxidative genes transcription [10]. Without stimulation, Nrf2 binds with two molecules of Keap-1 and is subsequently proteasomal degraded. Oxidative stressors or electrophiles dissociates the Nrf2 from Keap-1, prevents Nrf2 from degrading and increases nuclear accumulation of Nrf2. Also, Nrf2 has been shown to favor the progression, chemo- and radio-resistance of several types of cancers [11-13]. Previous studies have demonstrated that Nrf2 could directly inhibit the inflammatory cytokines transcription of immune cells and indirectly limit the inflammatory process through modulating HO-1 expression $[14,15]$. However, the role of Nrf2 in TAM formation and its function on cancer cell is unclear.

In this study, THP-1 or peripheral blood monocyte (PBMC) derived macrophages and cancer cells costimulation was performed to mimic the cancer cell and macrophages interaction in the tumor microenvironment. We found that Nrf2 was essential for driving macrophage activation into M2 phenotype by cancer cell stimulation and migration of cancer cell.

\section{Methods}

\section{Cell culture and treatment}

The hepatocellular carcinoma cell lines Hep G2 and Huh 7 were gotten from the Riken Cell Bank (Tsukuba, Japan). Pancreatic cancer cell lines Panc-1 was gotten from the Riken Cell Bank, and SUIT2 was gotten from Japanese Collection of Research Bio-resources Cell Bank (Ibaraki, Japan). These cell lines were passaged for fewer than 6 months after resuscitation. Cancer cells were cultured in DMEM (Life Technologies Japan Ltd., Tokyo, Japan) containing 10\% FBS (Life Technologies Japan Ltd., Tokyo, Japan). Human monocyte cell line, THP-1, gotten from Culture Collections of Public Health England, grew in RPMI-1640 (Wako, Osaka, Japan) containing 10\%FBS and $10 \mathrm{mM}$ HEPES. THP-1 cells were treated with $150 \mathrm{nM}$ phorbol-12-myristate-13-acetate (PMA) (Sigma, St. Louis, $\mathrm{MO}$, USA) for $48 \mathrm{~h}$ to induce to macrophages.

Cells were transfected with Nrf2 siRNA (Applied Biosystems, s9492, Waltham, Massachusetts, USA), Keap-1 siRNA (Applied Biosystems, s18981, Waltham, MA, USA) or negative control siRNA (Applied Biosystems, Select Negative Control \#1 siRNA, Waltham, MA, USA) at $10 \mathrm{nmol} / \mathrm{L}$ using Lipofectamine 2000 (Invitrogen, Thermo Fisher Scientific Inc., Waltham, MA, USA) according to the manufacturer's instructions.

\section{Conditioned medium (CM) of cells preparing}

The cells were washed with pre-warmed PBS twice, then incubated with the FBS-free medium. After $24 \mathrm{~h}$ of incubation, the medium was collected, centrifuged ( $450 \times \mathrm{g}, 5 \mathrm{mins}$ ) and filtrated by the $0.2 \mu \mathrm{m}$ filter. The conditioned medium was used without additional FBS supplemented.

\section{Migration assay}

The transwell inserts (Corning, NY. USA) with $8 \mu \mathrm{m}$ pore size were used for migration assay. $0.5-1 \times 10^{5}$ cancer cells were seeded in the upper chamber. After cell attached, the upper chambers were washed twice with DMEM gently, then the medium of upper chambers was changed to conditioned medium, and the same medium with 5\% FBS supplemented was used in the lower chamber. After $24 \mathrm{~h}$ incubation, the cells on the downside of transwell inserts were fixed in $4 \%$ paraformaldehyde and stained with $0.2 \%$ crystal violet. The stained cells in five random $\times 400$ microscopic fields were counted.

\section{Lactate assay}

The concentration of lactate in the conditioned medium was measured by using lactate assay kit (Sigma, St. Louis, MO, USA). The 10ul fresh conditioned medium was mixed with lactate assay buffer. Then 50ul of the master reaction mix was added to each sample. After incubating the reaction for $30 \mathrm{~min}$ at room temperature, measure the absorbance at $570 \mathrm{~nm}$.

\section{Reactive oxygen species (ROS) detection}

The cells were incubated with $2^{\prime}, 7^{\prime}$-Dichlorofluorescin diacetate (H2DCFDA $5 \mu \mathrm{M}$, Sigma) $30 \mathrm{~min}$. Then the cells were washed and received the stimulation of conditioned medium for $6 \mathrm{~h}$. The fluorescence intensity $(\lambda \mathrm{ex}=$ $495 / \lambda$ em $=529 \mathrm{~nm}$ ) was measured by the plate reader (SpectraMax i3, Molecular Devices, Tokyo, Japan).

\section{Enzyme-linked immunosorbent assay}

The level of IL-1, IL- 6 and VEGF were detected by using IL-1, IL-6 and VEGF Quantikine ELISA kit (R\&D Systems, Minneapolis, MN, USA) according to the manufacturer's protocol. Absorbance at $450 \mathrm{~nm}$ was measured using a plate reader as a correction wavelength of $540 \mathrm{~nm}$.

\section{Polymerase chain reaction}

The total RNA of each sample was extracted using the RNeasy Mini Kit (Qiagen, Hilden, Germany) according to the manufacturer's instructions. cDNA was synthesized using the reverse transcription kit (Applied Biosystems, Thermo Fisher Scientific Inc., Waltham, MA, USA). The following primers from TaqMan gene expression assays (assay identification number) were used: IL-1b (Hs01555410_m1); IL-6 (Hs00174131_m1); CD163 (Hs00174705_m1); Arg1 (Hs00163660_m1) and VEGF- $\alpha$ (Hs00163660_m1). GAPDH (4352339E) was selected as internal control. StepOnePlus Real-Time PCR System (Applied Biosystems, Thermo Fisher 
Scientific Inc., Waltham, MA, USA) was used to perform qRT-PCR.

\section{Western blotting}

Nuclear and cytoplasmic proteins were extracted by using NuCLEAR ${ }^{\mathrm{m}}$ Extraction Kit (Sigma, St. Louis, MO, USA). Total proteins were extracted by lysing cell with RIPA buffer (Thermo Fisher Scientific Inc., Waltham, MA, USA) containing the protease inhibitor cocktail (Sigma, St. Louis, MO, USA) and PhosSTOP phosphatase inhibitor cocktail (Roche, Tokyo, Japan). Then Proteins concentration were measured with the $\mathrm{BCA}$ kit (Thermo Fisher Scientific Inc., Waltham, MA, USA), and equal amounts of extracted proteins separated on 10\% SDS-PAGE gels and transferred onto PVDF membranes (Bio-Rad, Hercules, CA, USA). Membranes were incubated with the indicated primary antibody, then appropriate HRP-conjugated secondary antibody. The proteins were detected with Chemiluminescence (GE, Little Chalfont, Buckinghamshire, UK). The primary antibodies used in western blotting were listed in Table 1.

\section{Tumor cell transplantation}

Six to eight-week-old BALB/c nude mice were purchased from Charles River Company (Yokohama, Japan) and housed at the animal center of Tokushima University. All animal experiments were approved by Tokushima University Institutional Review Board. The experiments performed in accordance with the guidelines of the Animal Research Committee of Tokushima University Graduate School. To establish the animal model, 250ul of Panc-1 and Huh $7\left(2 \times 10^{7}\right.$ cells $\left./ \mathrm{ml}\right)$ containing $50 \%$ matrigel matrix (Corning, NY. USA) with or without educated macrophages $\left(2 \times 10^{5}\right.$ cells $)$ was injected subcutaneously. After four weeks, the mice were sacrificed and tumor tissues were collected.

\section{Immunochemistry}

The cells were washed with cold PBS and fixed with paraformaldehyde. Then the fixed cells were incubated with anti-Nrf2 primary antibody overnight at $4{ }^{\circ} \mathrm{C}$. Then after fluorophore-conjugated second antibody incubation, the cells were observed. The xenograft tumor tissues, which paraformaldehyde -fixed and paraffin-embedded, were incubated with primary monoclonal antibody CD163

Table 1 The first antibody used in western blot

\begin{tabular}{lll}
\hline Name & Product number & Company \\
\hline Histone H3 antibody & $\# 9715$ & Cell Signaling \\
b-Actin antibody & $\# 4967$ & Cell Signaling \\
N-cadherin antibody & $\# 13116$ & Cell Signaling \\
Nrf2 antibody & ab62352 & Abcam \\
E-cadherin antibody & ab1416 & Abcam \\
\hline
\end{tabular}

(ab87099, Abcam, Tokyo, Japan) and Nrf2 (ab62352, Abcam, Tokyo, Japan). After incubated with the fluorophore-conjected second antibody, the slides were checked by fluorescence microscope (Keyence Corporation, Itasca, IL, USA).

\section{Statistical analysis}

Data were presented as mean \pm SD. Statistical analyses were performed by SPSS 19.0 (IBM, NY, USA). The student $\mathrm{t}$-test was used to compare the difference between the two groups. One-way analysis of variance (one-way ANOVA) followed by Bonferroni' test was used to compare the differences more than two groups. There was a statistically significant difference when the $p$-value was less than 0.05 .

\section{Results}

\section{Cancer cells induced macrophages phenotype} transformation

After stimulated with PMA $48 \mathrm{~h}$, the suspended THP-1 attached the dishes and differentiated into macrophages (M0) [16]. Then the M0 macrophages were stimulated with CM of hepatocellular carcinoma cells (Hep G2 and Huh 7) and pancreatic carcinoma cells (Panc-1 and SUIT2) for another $24 \mathrm{~h}$ (Fig. 1a). After CM stimulation, the tumor educated macrophages (TEM) decreased the expression of M1 macrophage markers, such as IL-1b, IL-6 and increased the expression of M2 macrophage markers including Arg1 and CD163 compared with M0 macrophage. Also, the VEGF- $\alpha$ was up-regulated after education (Fig. 1b). As the same as THP-1 derived macrophages, the human peripheral blood monocyte (PBMC) derived macrophages increased M2 macrophage markers and VEGF expression after CM of cancer cells stimulation (Additional file 1: Figure S1A).

\section{Nrf2 activation correlated with M2 TEM formation and VEGF-a expression}

It has been reported that Nrf2 played the important role in the anti-inflammatory action [17]. Over-activation of Nrf2 could inhibit the pro-inflammatory cytokines expression [15]. In order to uncover whether the Nrf2 participated in the TEM phenotype transformation, Nrf2 expression of TEM was detected firstly. The immunofluorescent staining of Nrf2 demonstrated that the Nrf2 nuclear translocation of TEM apparently increased compared with M0 macrophages (Fig. 1c). Also, compared with the nuclear Nrf2 of M0, nuclear Nrf2 of TEM had an obviously higher level (Fig. 1d). HO-1 and Nqo-1, as the downstream genes of Nrf2, increased after cancer cell CM stimulation (Fig. 1e). The Nrf2 levels were also detected in PBMC derived TEM. Similar to THP-1 derived TEM, PBMC derived TEM also showed an increase of Nrf2 nuclear translocation (Additional file 1: Figure S1B-C). The 


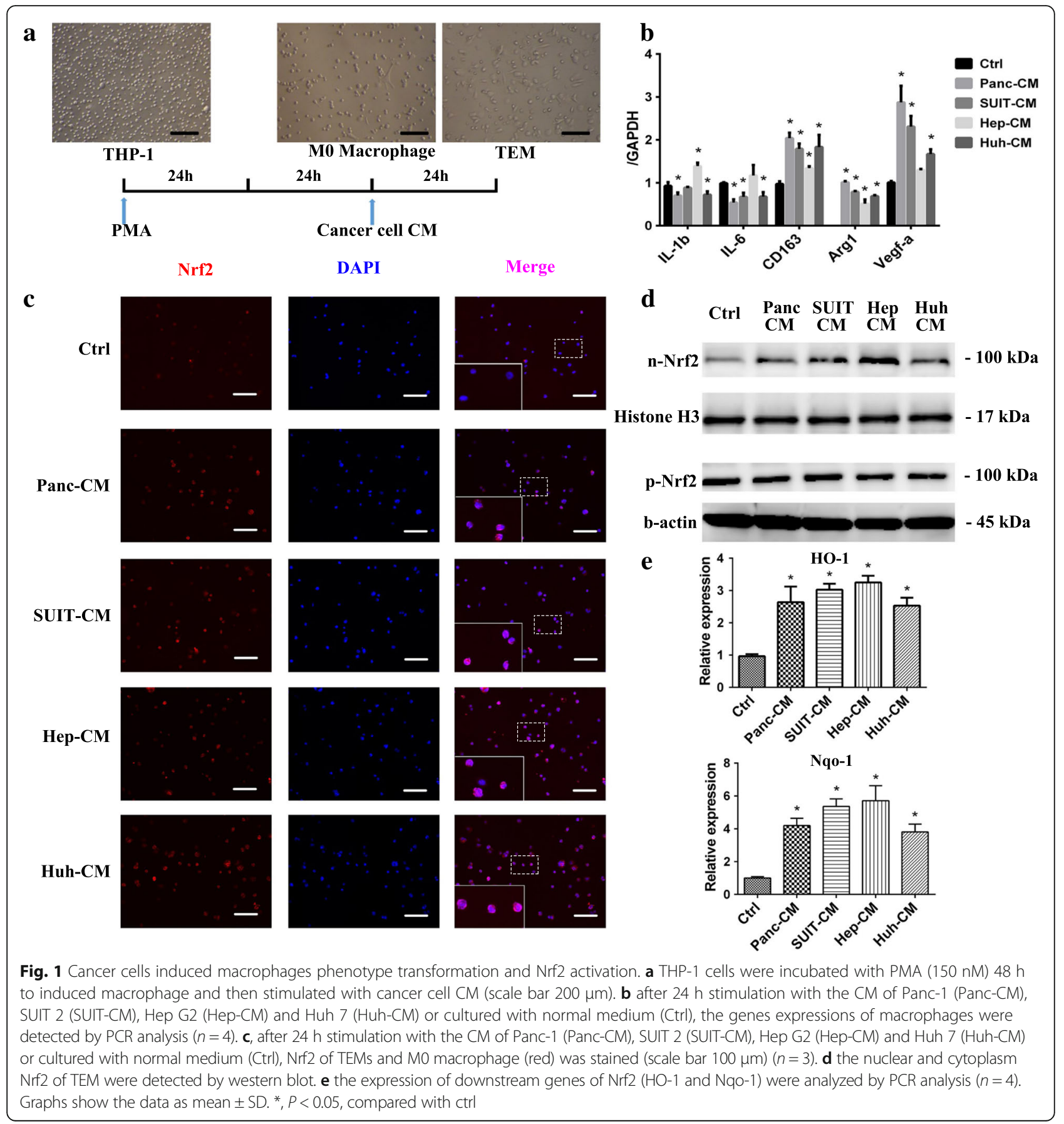

xenograft tumor tissues of cancer cells and THP-1 derived TEM co-injection and patients' tumor tissues showed that CD163-positive cells were also characterized as high Nrf2 nuclear-translocation (Additional file 1: Figure S1D-E). Above all, it has been demonstrated that Nrf2 was over-activated in the TEM by cancer cell CM stimulation.

To confirm the correlation between Nrf2 activation and TEM phenotype transformation, Nrf2 knocked-down of THP-1 derived M0 macrophage was performed by small interfering RNA treatment (Fig. 2a). The Nrf2 knocked- down was also verified by Nrf2 downstream genes expression (Additional file 1: Figure S2A). Nrf2 knock-down of macrophage canceled the effect of cancer cell $\mathrm{CM}$ which induced M1 macrophage markers down-regulation and M2 macrophage markers up-regulation (Fig. 2b-c and Additional file 1: Figure S2B). Moreover, Nrf2 knockdown diminished the cancer cell CM-induced VEGF- $\alpha$ expression and secretion (Fig. 2b-c).

Keap-1 mediated Nrf2 degradation is the dominating pathway to modulate Nrf2 activation [18]. When the cell 


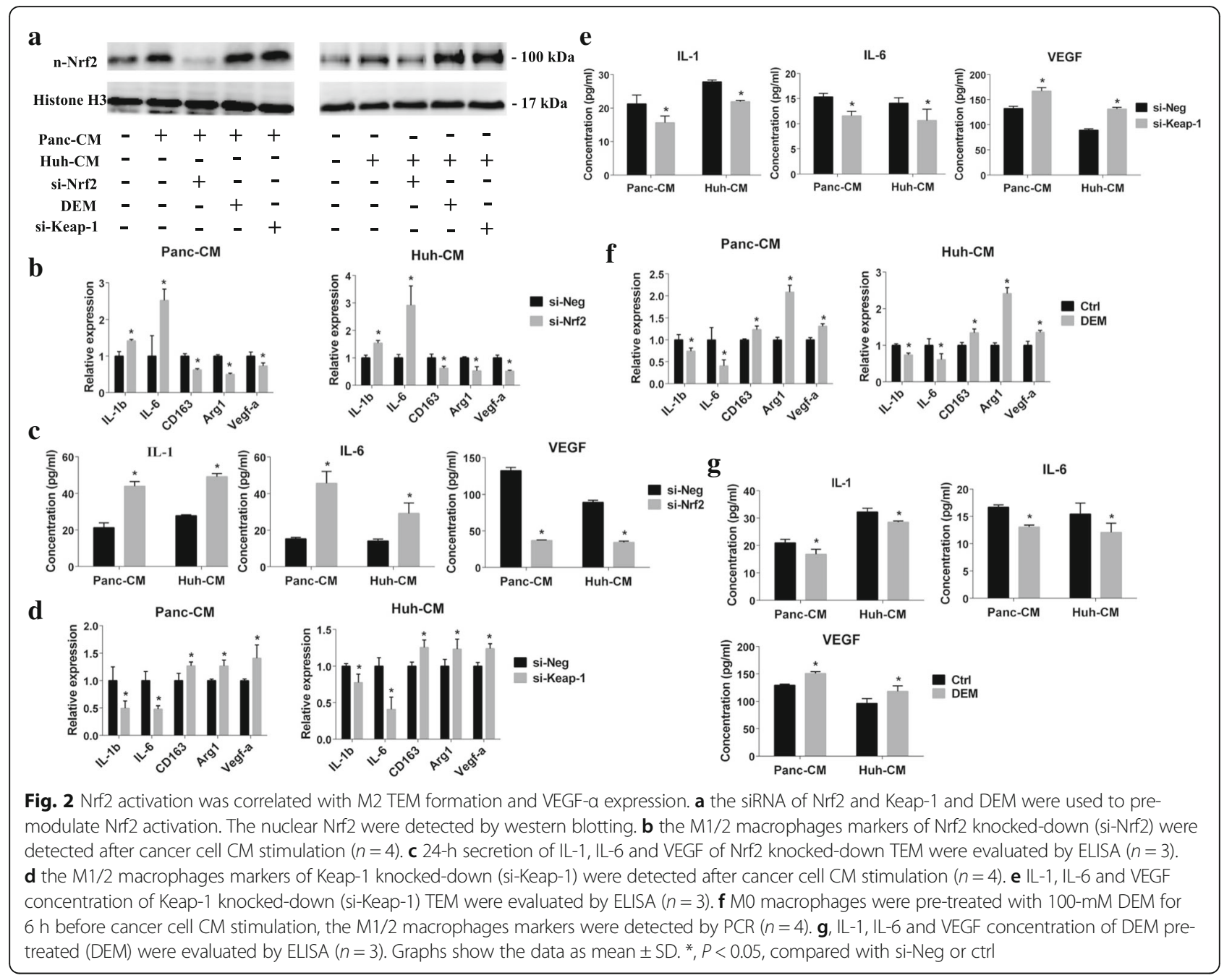

receives stimulation, Nrf2-Keap-1 complex disassembles and Nrf2 translocates into nuclear. Keap-1 knocked-down was utilized to manipulate the $\mathrm{Nrf} 2$ activation. Increased Nrf2 nuclear accumulation and Nrf2-dependent transcription were confirmed after Keap-1 knocked-down (Fig. 2a and Additional file 1: Figure S2A). Keap-1 knocked-down amplified M2-macrophages induction by cancer cells, appearing as decreased IL-1b and IL-6 expression, and increased CD163 and Arg1 expression (Fig. 2d-e and Additional file 1: Figure S2B). The application of Diethyl maleate (DEM), a small molecule Nrf2 activator, was also confirmed that Nrf2 activation of macrophages enhanced cancer cell-induced M2 macrophages transformation and VEGF- $\alpha$ expression [15] (Fig. 2f-g and Additional file 1: Figure S2B).

\section{Lactate secreted by cancer cell promoted TEM Nrf2 activation}

Our data revealed that the cancer cell CM activated Nrf2 in macrophage correlated with M2 features of TEM, we next sought to identify the tumor-derived soluble factor(s) which was capable of Nrf2 activation. Firstly, to determine the nature of cancer cell secreted factors that alter macrophage subtype, the cancer cell conditioned medium was subjected to three freeze-thaw cycles $\left(-80{ }^{\circ} \mathrm{C}, 60^{\circ} \mathrm{C}\right)$. We found that $\mathrm{CM}$ of the cancer cell with or without processing both could increase M2 feature of TEM indicating that the factor(s) lacked tertiary structure (Fig. 3a). It meant that proteins which achieved functions relying on the tertiary and quaternary structure were excluded [19]. So, small molecule metabolites could be the candidates. The well-known Warburg effect demonstrates that lactate would be highly produced by cancer cell through aerobic glycolysis even at normoxia condition [20]. Oxalic acid was the one of LDH inhibitor which converted pyruvate to lactate [21]. To verify whether lactate in cancer cell CM correlated with the Nrf2 activation of TEM, we reduced the lactate in cancer cell CM by pre-incubated the cancer cell with the oxalic acid containing media (Fig. 3b). It showed that the lactate-reduced CM of cancer cells lost the capability of promoting $\mathrm{Nrf} 2$ activation of macrophages 


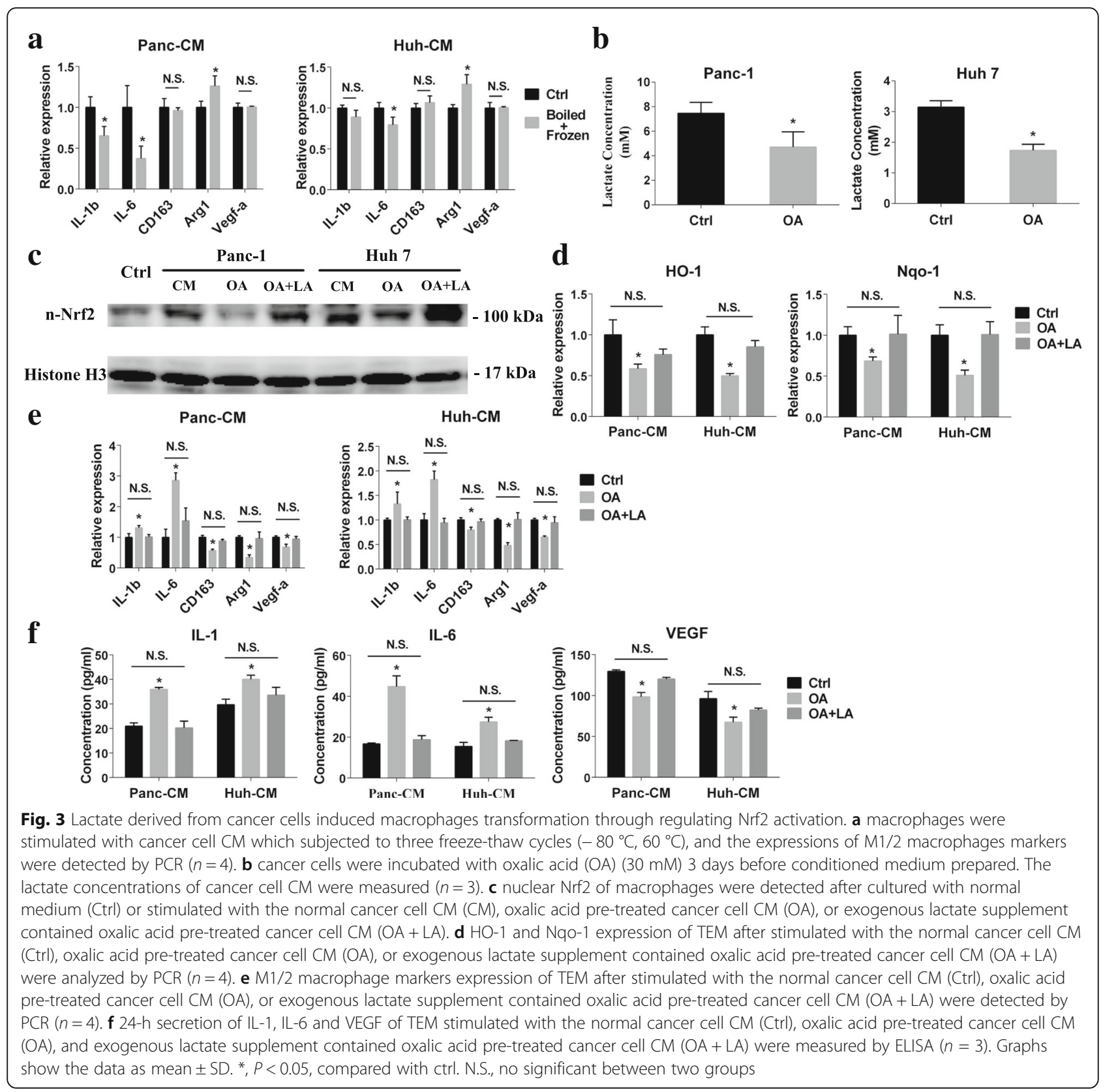

which could be rescued by exogenous lactate supplement (3 mM) (Fig. 3c-d). However, the exogenous hydrochloric acid which could also decrease the $\mathrm{pH}$ value as lactate could not promote the Nrf2 activation of macrophages (Data were not showed). Concomitantly, lactate-reduced $\mathrm{CM}$ of cancer cell failed to induce TEM phenotype transformation until exogenous lactate added (Fig. 3e-f and Additional file 1: Figure S3A-B).

\section{ROS formation regulated by lactate activated Nrf2}

In the previous results, we confirmed that the lactate derived from the cancer cell activated the Nrf2 of TEM and induced its M2 feature. However, the detail of the Nrf2 activation of TEM by lactate is unknown. It had been reported that lactate stimulation could increase the intercellular ROS [22]. In our study, the fluorescence intensity of $2^{\prime}, 7^{\prime}$-dichlorofluorescein (DCF) showed that normal cancer cell CM significantly up-regulated intracellular ROS level of macrophage comparing with the lactate reduced ones or without cancer cell CM stimulation (Fig. 4a). Also, as Fig. 4a showed, exogenous lactate could recover the ROS level as normal cancer cell $\mathrm{CM}$-induced. Above all, it confirmed that the lactate in the cancer cell $\mathrm{CM}$ was responsible for ROS formation in TEM. In the canonical pathways, Nrf2 activation is responsible for ROS increase [23]. In order to uncover 


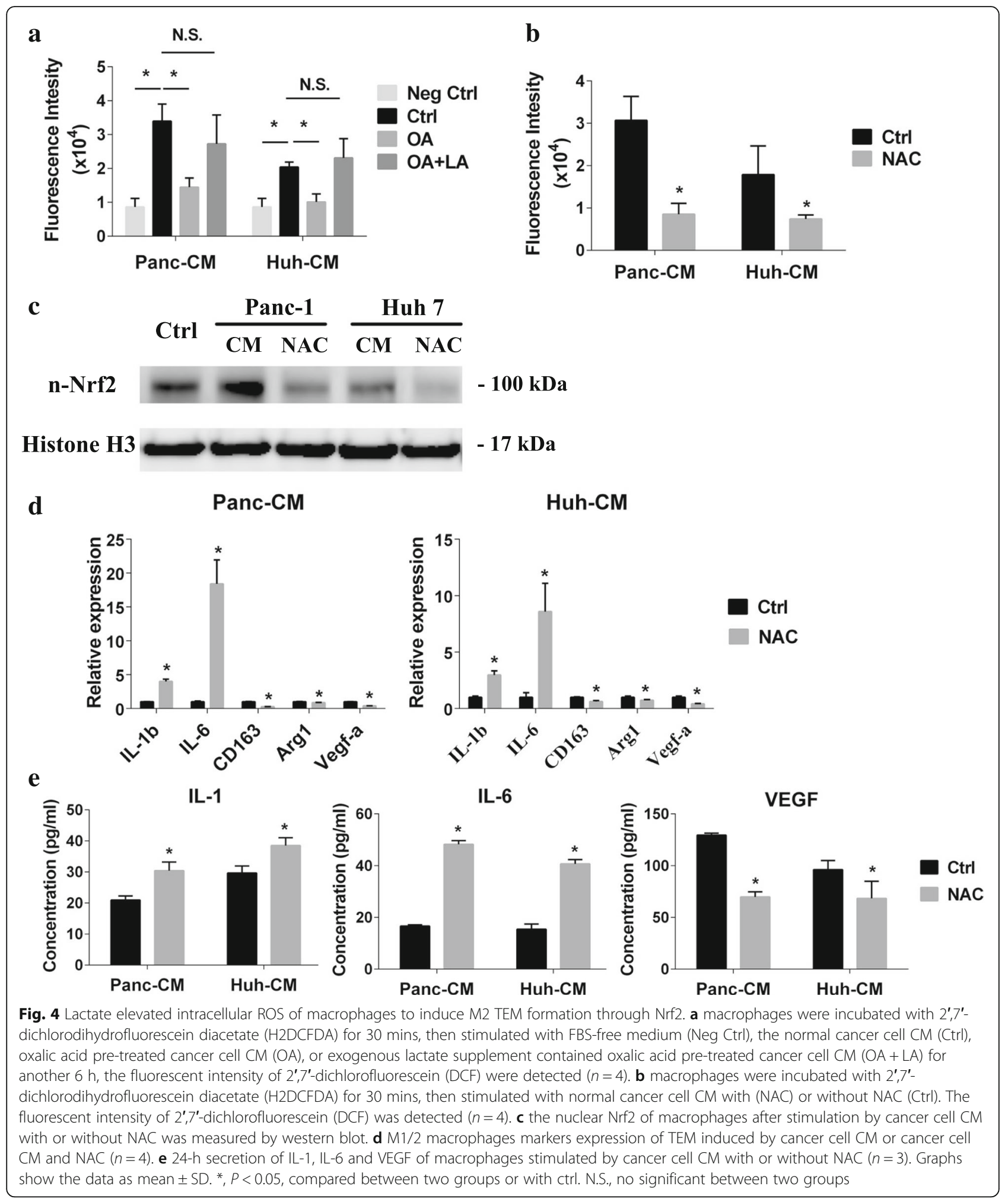

whether Nrf2 activation in TEM relied on lactate-induced ROS or not, we inhibited the ROS formation in macrophages during cancer cell CM stimulation by NAC treatment (Fig. 4b). NAC treatment canceled the Nrf2 nuclear accumulation which appeared after cancer cell CM stimulation (Fig. 4c). Moreover, ROS inhibition diminished the macrophage phenotype remodeling (Fig. 4d-e and Additional file 1: Figure S3C-D). 
TEM activated Nrf2 of cancer cells to promote cancer cell EMT

In the CD163 and Nrf2 double staining of mice xenograft tumor tissues, the Nrf2 nuclear translocation could be observed not only in CD163-positive cells but also in the cancer cells around the CD163 positive macrophages (Fig. 5a). In the patients' tumor tissues, the nuclear Nrf2 positive cancer cells could also observe near the CD163 positive cells (Additional file 1: Figure S4A). In order to uncover that whether the Nrf2 activation of cancer cells correlated with CD163-positive macrophages stimulation or not, we stimulated cancer cells with CM of TEM or M0 macrophages in vitro. The higher Nrf2 nuclear accumulation of cancer cell was detected after stimulation with CM of TEM both derived from THP-1 or PBMC (Fig. 5b and Additional file 1: Figure S4B). It has been reported that Nrf2 activation could promote cancer metastasis by modulating epithelial-mesenchymal transition (EMT) [24]. Nrf2 knocked-down in cancer cells before TEM CM stimulation was performed to illustrate the relationship between TEM-induced cancer cell Nrf2 activation and EMT (Fig. 5c-d and Additional file 1: Figure. S4C-D). The migration assay showed that TEM CM significantly increased the cancer cell migration compared with M0-CM. However, Nrf2 knocked-down of cancer cell blunted the TEM migration-promoting effect (Fig. 5e and Additional file 1: Figure S4E). After TEM CM stimulation, the morphological changes of cancer cells resembling EMT were clearly observed on normal cancer cells but not on Nrf2 knocked-down ones, with cells becoming spindle-shaped and scattered compared to cobble stone-shaped (Fig. $5 \mathrm{f}$ and Additional file 1: Figure S4F). Moreover, the EMT related markers expression revealed that TEM promoted cancer cell EMT through Nrf2 activation, as Nrf2 knocked-down diminished the TEM modulated EMT expression (Fig. $5 \mathrm{~g}$ and Additional file 1: Figure S4G).

\section{TEM derived VEGF stimulated cancer cell EMT through Nrf2 activation}

Based on the previous report, VEGF could increase cancer cell proliferation, migration ability [25]. Considering that previous results showed M0 macrophages and Nrf2 knock-down TEM, both of which had lower VEGF expression than normal TEM, partly lost cancer cell migration-promoting ability, VEGF might be a candidate responsible for TEM induced cancer cell EMT. VEGF neutralization by the anti-VEGF antibody (R\&D Systems, Minneapolis, MN, USA) canceled the cancer cell migration-promoting effect of TEM CM was detected (Fig. 6a and Additional file 1: Figure S5A). The morphological changes as characteristic of EMT vanished when the VEGF was neutralized (Fig 6b and Additional file 1: Figure S5B). The EMT markers expression of cancer cell also showed that VEGF reduction attenuated the EMT promoting-effect of educated macrophage (Fig. 6c and Additional file 1: Figure S5C). Also, VEGF neutralization in educated macrophage conditioned medium could not induce the Nrf2 activation and Nrf2-dependent transcription (Fig. 6d-e and Additional file 1: Figure S5D-E). Above all, it demonstrated that TEM derived VEGF stimulated cancer cell EMT through Nrf2 activation.

\section{Discussion}

In this study, we described a new role of Nrf2, relevant for cancer-induced macrophages phenotype transformation and paracrine action of TAM on cancer cell EMT. Our study demonstrated the existence of a cross-talk between macrophages and cancer cells. Cancer cells secreted lactate, which elevated ROS in macrophages, induced macrophage M2 phenotype transformation and VEGF expression through Nrf2 mediation. On the other hand, cancer cell educated macrophages promoted cancer cell migration partially relied on the increased Nrf2 activation of cancer cell by VEGF secretion (Fig. 6f).

Peripheral blood monocytes are recruited and activated to form a wide spectrum of TAM in response to chemokines and growth factors to construct the tumor microenvironment [2]. In the tumor microenvironments, there are not only IFN- $\gamma$, TNF-a, and GM-CSF which could activate macrophages like M1 macrophages, but also IL-4, IL-10 and CSF-1 which induce M2 macrophages differentiation [26]. In our study, the cancer cells provoked M2 phenotype as demonstrated by an increase of CD163 and Arg1 and a decrease of IL-1b and IL-6 expression. The $\mathrm{CD}_{163^{+}}$or M2 macrophage, as a prognosis factor, induce the cancer progression including cancer cell proliferation, migration and invasion, and angiogenesis [5, 27, 28].

$\mathrm{Nrf2}$, a key regulator for the maintenance of redox homeostasis, has been demonstrated to contribute to cell proliferation and malignant phenotypes [29, 30]. Previous decades, the role of Nrf2 in immune modulation have been recognized. As our study showed Nrf2 activation in macrophages inhibited the IL-1b and IL- 6 expression, it has been demonstrated that activation of Nrf2 prevented LPS-induced upregulation of pro-inflammatory cytokines, including IL-1b and IL-6 [15]. IL-1b and IL-6 production are increased in $\mathrm{Nrf}^{-/-}$mice with dextran sulfate-induced colitis [31]. Moreover, Nrf2 could affect macrophage polarization toward the M2 phenotype through its downstream genes HO-1 [32]. Consistent with our study, Nrf2 activation in macrophage increased M2 markers including CD163 and Arg1 expression. However, some reports showed that Nrf2-deficient myeloid lineages but not Nrf2 wild-type could increase lung cancer metastasis in vivo $[33,34]$. In these studies, the Nrf2 of 


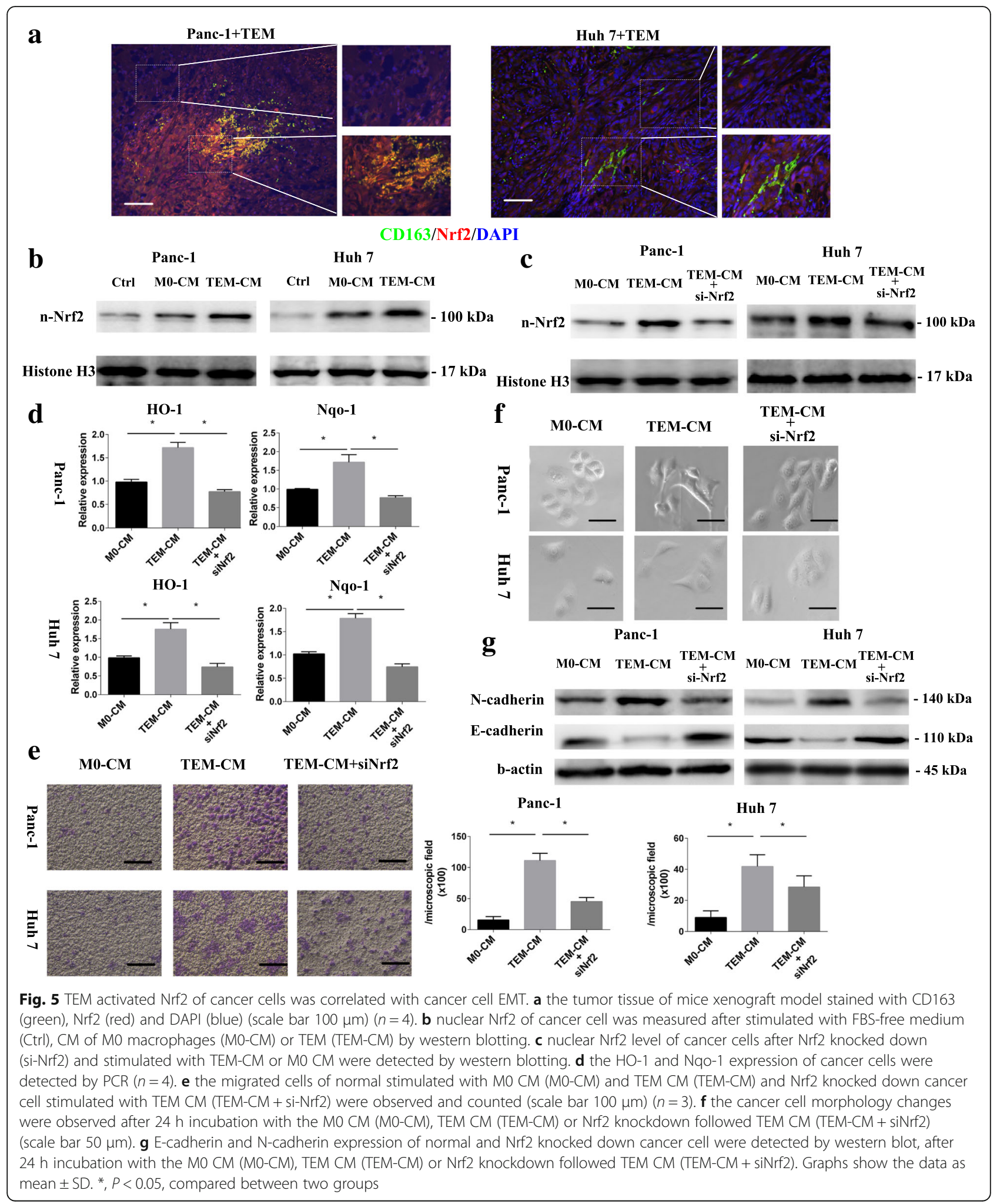

myeloid-derived cells but not macrophage were modulated. It might be related with the pro-differentiation action of Nrf2 on myeloid lineages which affect anti-tumor immune cell formation [35].
The Warburg effect widely exists among the cancer cells provide cancer cell production for nucleotide, amino acids and lipids for proliferation [36, 37]. The Warburg effect causes increased production of lactate which could not 


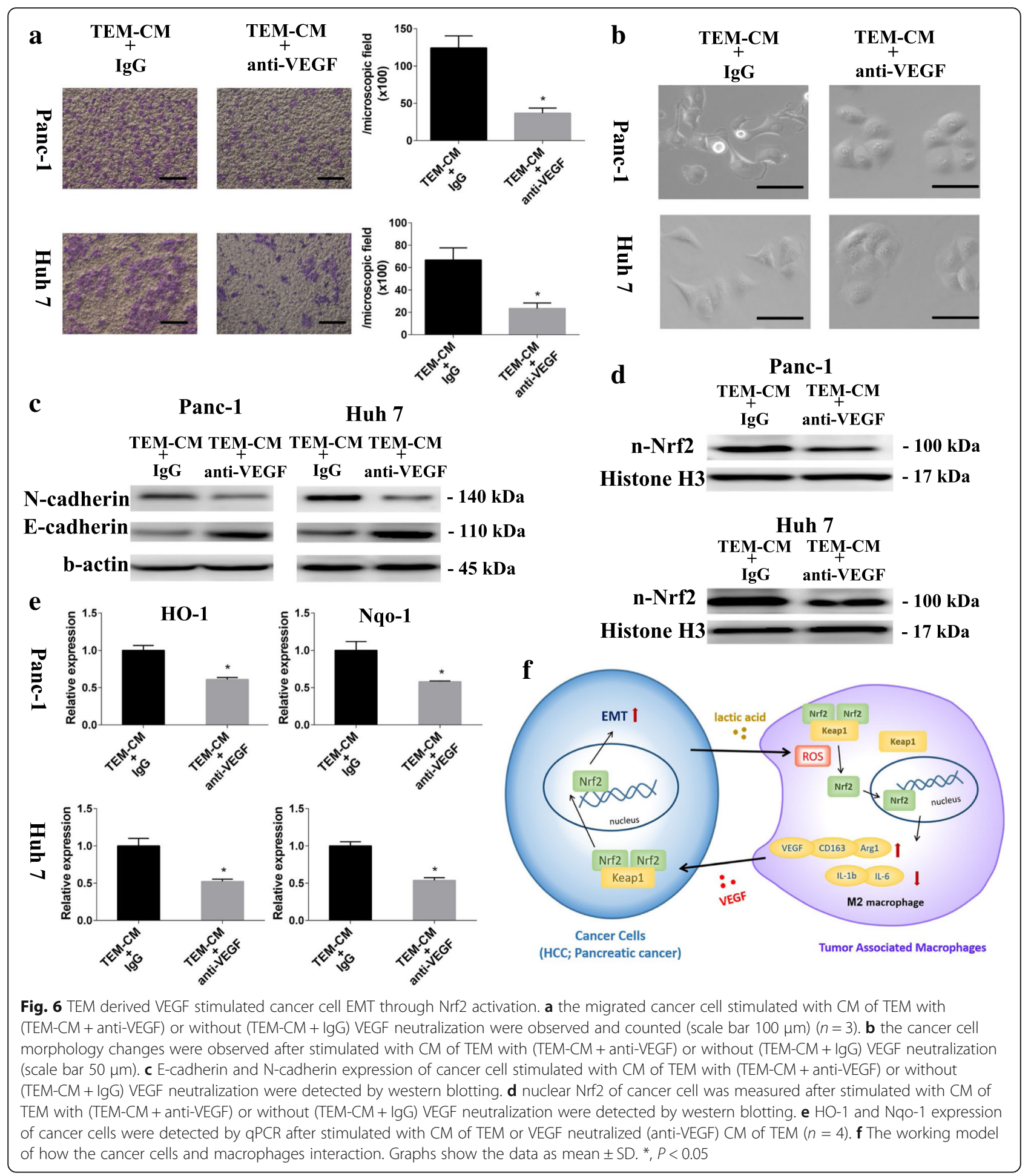

only regulate the $\mathrm{pH}$ level but also modulate the inflammation of the tumor microenvironment $[38,39]$. Lactate can suppress TLR-4 mediated inflammation in the liver and pancreatic tissue [40]. Also, it has been reported that lactate induces M2 like macrophages through HIF-1a mediation [41]. G protein-coupled receptor 132 is a stress-inducible transmembrane receptor that responses to lactate but not the acidic condition related to M2 macrophage transformation by Nrf2 activation [42]. Although no certain study was reported the relation between lactate and Nrf2, there is the indirect evidence in which lactate has been proved to elevate the intercellular ROS, 
as a Nrf2 activator, or HIF-1a which is usually reciprocal activation of Nrf2 show the clues of the relationship of lactate and Nrf2 [43].

Our results also implied that the Nrf2 over-activation in the tumor was necessarily associated with TAM promote migration through induced EMT. The association of Nrf2 and cancer malignancy has been previously shown. The previous analyses of Nrf2 expression or activation in human tumors have revealed that high Nrf2 expression or activation correlated with poor prognosis in hepatocellular carcinoma and pancreatic cancer [44, 45]. Actually, Nrf2 not only maintains cancer cell growth at oxidative stress, but also increase the cancer cell invasion potential through downregulated E-cadherin expression [13, 46].

The effect of VEGF on cancer cell migration and invasion has been reported decades ago [47]. With the anti-VEGFR treatment wildly performed in the clinical, VEGF-VEGFR induced cancer metastasis is further noticed. The association of VEGF and Nrf2 activation expression has been reported in Kweider's study in which VEGF activated the Nrf2 in an ERK1/2-dependent manner [48]. Hypoxia due to Nrf2 activation can regulate HIF-1 $\alpha$ /VEGF signaling pathways to modulate angiogenesis [49]. In addition, Nrf2 knockdown significantly decreased VEGF expression [50]. Our results demonstrated that TEM derived VEGF could activate Nrf2 activation in the cancer cell.

\section{Conclusions}

In conclusion, lactate derived from cancer cells stimulate macrophages M2 phenotype transformation through Nrf2 activation. TEM secrete VEGF to promote cancer cell migration by Nrf2 induced EMT. These findings not only cover the cancer cell and macrophages interaction to contribute to tumor progression but also provides comprehensive insights into the role of Nrf2 in TAM formation and tumor metastasis.

\section{Additional file}

Additional file 1: Supplement methods and figures. (PDF $6318 \mathrm{~kb}$ )

\begin{abstract}
Abbreviations
CM: Conditioned medium; DCF: 2',7'-dichlorofluorescein; DMEM: Dulbecco's modified eagle medium; EMT: Epithelial-mesenchymal transition; FBS: Fetal bovine serum; H2DCFDA: 2', 7'-Dichlorofluorescin diacetate; HCC: Hepatocellular carcinoma; Nrf2: Nuclear factor (erythroid-derived 2)-like 2; PBMC: Peripheral blood monocyte; PMA: Phorbol-12-myristate-13-acetate; ROS: Reactive oxygen species; TAM: Tumor-associated macrophages; TEM: Tumor educated macrophage
\end{abstract}

\section{Availability of data and materials}

Data sharing is not applicable to this article as no datasets were generated or analyzed during the current study.

\section{Authors' contributions}

RF designed the experiment and acquired the data and drafted the article. TI and YS analyzed and interpreted the data. SI and Shl acquired the data. YM revised the manuscript. YM and MS designed and instructed the experiment and made the final approval of the version to be published. All authors read and approved the final manuscript.

\section{Ethics approval and consent to participate}

All animal experiments were approved by Tokushima University Institutional Review Board. The experiments performed in accordance with the guidelines of the Animal Research Committee of Tokushima University Graduate School. The patients whose tissue sample were used in this study and the health donor who provided the blood in this study provided written informed consent. The study protocol was approved by the Institutional Review Board of our center.

\section{Consent for publication}

Not applicable

\section{Competing interests}

The authors declare that they have no competing interests.

\section{Publisher's Note}

Springer Nature remains neutral with regard to jurisdictional claims in published maps and institutional affiliations.

Received: 12 July 2018 Accepted: 14 August 2018

Published online: 04 September 2018

\section{References}

1. Quail DF, Joyce JA. Microenvironmental regulation of tumor progression and metastasis. Nat Med. 2013;19:1423-37.

2. Franklin RA, Liao W, Sarkar A, Kim MV, Bivona MR, Liu K, Pamer EG, Li MO. The cellular and molecular origin of tumor-associated macrophages. Science. 2014;344:921-5.

3. Franklin RA, Li MO. Ontogeny of tumor-associated macrophages and its implication in Cancer regulation. Trends Cancer. 2016;2:20-34.

4. Komohara Y, Fujiwara Y, Ohnishi K, Takeya M. Tumor-associated macrophages: potential therapeutic targets for anti-cancer therapy. Adv Drug Deliv Rev. 2016;99:180-5.

5. Hu H, Hang JJ, Han T, Zhuo M, Jiao F, Wang LW. The M2 phenotype of tumor-associated macrophages in the stroma confers a poor prognosis in pancreatic cancer. Tumour Biol. 2016;37:8657-64.

6. Edin S, Wikberg ML, Dahlin AM, Rutegard J, Oberg A, Oldenborg PA, Palmqvist $R$. The distribution of macrophages with a M1 or M2 phenotype in relation to prognosis and the molecular characteristics of colorectal cancer. PLoS One. 2012;7:e47045.

7. Dong P, Ma L, Liu L, Zhao G, Zhang S, Dong L, Xue R, Chen S. CD86(+ )/CD206(+), diametrically polarized tumor-associated macrophages, Predict Hepatocellular Carcinoma Patient Prognosis. Int J Mol Sci. 2016;17:320.

8. Ma R, Ji T, Chen D, Dong W, Zhang H, Yin X, Ma J, Liang X, Zhang Y, Shen $\mathrm{G}$, et al. Tumor cell-derived microparticles polarize M2 tumor-associated macrophages for tumor progression. Oncoimmunology. 2016;5:e1118599.

9. Chen W, Ma T, Shen XN, Xia XF, Xu GD, Bai XL, Liang TB. Macrophageinduced tumor angiogenesis is regulated by the TSC2-mTOR pathway. Cancer Res. 2012;72:1363-72.

10. Kensler TW, Wakabayashi N, Biswal S. Cell survival responses to environmental stresses via the Keap1-Nrf2-ARE pathway. Annu Rev Pharmacol Toxicol. 2007:47:89-116.

11. Jeong Y, Hoang NT, Lovejoy A, Stehr H, Newman AM, Gentles AJ, Kong W, Truong D, Martin S, Chaudhuri A, et al. Role of KEAP1/NRF2 and TP53 mutations in lung squamous cell carcinoma development and radiation resistance. Cancer Discov. 2017;7:86-101.

12. Zavattari P, Perra A, Menegon S, Kowalik MA, Petrelli A, Angioni MM, Follenzi A, Quagliata L, Ledda-Columbano GM, Terracciano L, et al. Nrf2, but not beta-catenin, mutation represents an early event in rat hepatocarcinogenesis. Hepatology. 2015;62:851-62.

13. Chio II, Jafarnejad SM, Ponz-Sarvise M, Park Y, Rivera K, Palm W, Wilson J, Sangar V, Hao Y, Ohlund D, et al. NRF2 promotes tumor maintenance by modulating mRNA translation in pancreatic Cancer. Cell. 2016;166:963-76.

14. Bolisetty S, Zarjou A, Hull TD, Traylor AM, Perianayagam A, Joseph R, Kamal Al, Arosio P, Soares MP, Jeney $V$, et al. Macrophage and epithelial cell $\mathrm{H}$ ferritin expression regulates renal inflammation. Kidney Int. 2015;88:95-108.

15. Kobayashi EH, Suzuki T, Funayama R, Nagashima T, Hayashi M, Sekine H, Tanaka N, Moriguchi T, Motohashi H, Nakayama K, Yamamoto M. Nrf2 
suppresses macrophage inflammatory response by blocking proinflammatory cytokine transcription. Nat Commun. 2016;7:11624.

16. Zhang J, Zhang Q, Lou Y, Fu Q, Chen Q, Wei T, Yang J, Tang J, Wang J, Chen Y, et al. HIF-1alpha/lL-1 beta signaling enhances hepatoma epithelialmesenchymal transition via macrophages in a hypoxic-inflammatory microenvironment. Hepatology. 2017;

17. Ahmed SM, Luo L, Namani A, Wang XJ, Tang X. Nrf2 signaling pathway: pivotal roles in inflammation. Biochim Biophys Acta. 1863;2017:585-97.

18. Suzuki T, Maher J, Yamamoto M. Select heterozygous Keap1 mutations have a dominant-negative effect on wild-type Keap1 in vivo. Cancer Res. 2011;71: $1700-9$

19. Sousa CM, Biancur DE, Wang $X$, Halbrook CJ, Sherman MH, Zhang L, Kremer D, Hwang RF, Witkiewicz AK, Ying H, et al. Pancreatic stellate cells support tumour metabolism through autophagic alanine secretion. Nature. 2016;536: 479-83

20. Romero-Garcia S, Moreno-Altamirano MM, Prado-Garcia H, Sanchez-Garcia FJ. Lactate contribution to the tumor microenvironment: mechanisms, Effects on Immune Cells and Therapeutic Relevance. Front Immunol. 2016;7:52.

21. Zhao Z, Han F, Yang S, Wu J, Zhan W. Oxamate-mediated inhibition of lactate dehydrogenase induces protective autophagy in gastric cancer cells: involvement of the Akt-mTOR signaling pathway. Cancer Lett. 2015;358:17-26.

22. Luo ST, Zhang DM, Qin Q, Lu L, Luo M, Guo FC, Shi HS, Jiang L, Shao B, Li $M$, et al. The promotion of erythropoiesis via the regulation of reactive oxygen species by lactic acid. Sci Rep. 2017;7:38105.

23. Nguyen T, Nioi P, Pickett CB. The Nrf2-antioxidant response element signaling pathway and its activation by oxidative stress. J Biol Chem. 2009;284:13291-5.

24. Kim WD, Kim YW, Cho IJ, Lee CH, Kim SG. E-cadherin inhibits nuclear accumulation of Nrf2: implications for chemoresistance of cancer cells. J Cell Sci. 2012;125:1284-95.

25. Luo M, Hou L, Li J, Shao S, Huang S, Meng D, Liu L, Feng L, Xia P, Qin T, Zhao X. VEGF/NRP-1axis promotes progression of breast cancer via enhancement of epithelial-mesenchymal transition and activation of NFkappaB and beta-catenin. Cancer Lett. 2016;373:1-11.

26. Caronni N, Savino B, Bonecchi R. Myeloid cells in cancer-related inflammation. Immunobiology. 2015;220:249-53

27. Yeung OW, Lo CM, Ling CC, Qi X, Geng W, Li CX, Ng KT, Forbes SJ, Guan XY, Poon RT, et al. Alternatively activated (M2) macrophages promote tumour growth and invasiveness in hepatocellular carcinoma. J Hepatol. 2015;62: $607-16$

28. Takeda Y, Costa S, Delamarre E, Roncal C, Leite de Oliveira R, Squadrito ML, Finisquerra V, Deschoemaeker S, Bruyere F, Wenes M, et al. Macrophage skewing by Phd2 haplodeficiency prevents ischaemia by inducing arteriogenesis. Nature. 2011;479:122-6.

29. DeNicola GM, Karreth FA, Humpton TJ, Gopinathan A, Wei C, Frese K, Mangal D, Yu KH, Yeo CJ, Calhoun ES, et al. Oncogene-induced Nrf2 transcription promotes ROS detoxification and tumorigenesis. Nature. 2011:475:106-9.

30. Mitsuishi Y, Taguchi K, Kawatani Y, Shibata T, Nukiwa T, Aburatani H, Yamamoto M, Motohashi H. Nrf2 redirects glucose and glutamine into anabolic pathways in metabolic reprogramming. Cancer Cell. 2012;22:66-79.

31. Khor TO, Huang MT, Kwon KH, Chan JY, Reddy BS, Kong AN. Nrf2-deficient mice have an increased susceptibility to dextran sulfate sodium-induced colitis. Cancer Res. 2006;66:11580-4.

32. Jadhav A, Tiwari S, Lee P, Ndisang JF. The heme oxygenase system selectively enhances the anti-inflammatory macrophage-M2 phenotype, reduces pericardial adiposity, and ameliorated cardiac injury in diabetic cardiomyopathy in Zucker diabetic fatty rats. J Pharmacol Exp Ther. 2013:345:239-49.

33. Hiramoto K, Satoh H, Suzuki T, Moriguchi T, Pi J, Shimosegawa T, Yamamoto M. Myeloid lineage-specific deletion of antioxidant system enhances tumor metastasis. Cancer Prev Res (Phila). 2014;7:835-44.

34. Satoh H, Moriguchi T, Taguchi K, Takai J, Maher JM, Suzuki T, Winnard PT Jr, Raman V, Ebina M, Nukiwa T, Yamamoto M. Nrf2-deficiency creates a responsive microenvironment for metastasis to the lung. Carcinogenesis. 2010:31:1833-43

35. Bobilev I, Novik V, Levi I, Shpilberg O, Levy J, Sharoni Y, Studzinski GP, Danilenko M. The Nrf2 transcription factor is a positive regulator of myeloid differentiation of acute myeloid leukemia cells. Cancer Biol Ther. 2011;11: $317-29$.
36. Warburg $\mathrm{O}$, Wind F, Negelein E. The metabolism of tumors in the body. J Gen Physiol. 1927:8:519-30

37. Vander Heiden MG, Cantley LC, Thompson CB. Understanding the Warburg effect: the metabolic requirements of cell proliferation. Science. 2009;324: 1029-33.

38. Xie H, Hanai J, Ren JG, Kats L, Burgess K, Bhargava P, Signoretti S, Billiard J, Duffy KJ, Grant A, et al. Targeting lactate dehydrogenase--a inhibits tumorigenesis and tumor progression in mouse models of lung cancer and impacts tumor-initiating cells. Cell Metab. 2014;19:795-809.

39. Husain Z, Huang Y, Seth P, Sukhatme VP. Tumor-derived lactate modifies antitumor immune response: effect on myeloid-derived suppressor cells and NK cells. J Immunol. 2013;191:1486-95.

40. Hoque R, Faroog A, Ghani A, Gorelick F, Mehal WZ. Lactate reduces liver and pancreatic injury in toll-like receptor- and inflammasome-mediated inflammation via GPR81-mediated suppression of innate immunity. Gastroenterology. 2014;146:1763-74.

41. Colegio OR, Chu NQ, Szabo AL, Chu T, Rhebergen AM, Jairam V, Cyrus N, Brokowski CE, Eisenbarth SC, Phillips GM, et al. Functional polarization of tumour-associated macrophages by tumour-derived lactic acid. Nature. 2014;513:559-63.

42. Chen P, Zuo H, Xiong H, Kolar MJ, Chu Q, Saghatelian A, Siegwart DJ, Wan Y. Gpr132 sensing of lactate mediates tumor-macrophage interplay to promote breast cancer metastasis. Proc Natl Acad Sci U S A. 2017;114:580-5.

43. Malec V, Gottschald OR, Li S, Rose F, Seeger W, Hanze J. HIF-1 alpha signaling is augmented during intermittent hypoxia by induction of the Nrf2 pathway in NOX1-expressing adenocarcinoma A549 cells. Free Radic Biol Med. 2010;48:1626-35.

44. Sun $X$, Ou Z, Chen R, Niu X, Chen D, Kang R, Tang D. Activation of the p62-Keap1-NRF2 pathway protects against ferroptosis in hepatocellular carcinoma cells. Hepatology. 2016;63:173-84.

45. Soini Y, Eskelinen M, Juvonen P, Karja V, Haapasaari KM, Saarela A, Karihtala P. Nuclear Nrf2 expression is related to a poor survival in pancreatic adenocarcinoma. Pathol Res Pract. 2014:210:35-9.

46. Arfmann-Knubel S, Struck B, Genrich G, Helm O, Sipos B, Sebens S, Schafer $H$. The crosstalk between Nrf2 and TGF-beta1 in the epithelial-mesenchymal transition of pancreatic duct epithelial cells. PLoS One. 2015;10:e0132978.

47. Wey JS, Fan F, Gray MJ, Bauer TW, McCarty MF, Somcio R, Liu W, Evans DB, Wu Y, Hicklin DJ, Ellis LM. Vascular endothelial growth factor receptor-1 promotes migration and invasion in pancreatic carcinoma cell lines. Cancer. 2005; 104:427-38.

48. Kweider N, Fragoulis A, Rosen C, Pecks U, Rath W, Pufe T, Wruck CJ. Interplay between vascular endothelial growth factor (VEGF) and nuclear factor erythroid 2-related factor-2 (Nrf2): implications for preeclampsia. J Biol Chem. 2011;286:42863-72

49. Li L, Pan H, Wang H, Li X, Bu X, Wang Q, Gao Y, Wen G, Zhou Y, Cong Z, et al. Interplay between VEGF and Nrf2 regulates angiogenesis due to intracranial venous hypertension. Sci Rep. 2016;6:37338.

50. Kuang L, Feng J, He G, Jing T. Knockdown of Nrf2 inhibits the angiogenesis of rat cardiac micro-vascular endothelial cells under hypoxic conditions. Int J Biol Sci. 2013:9:656-65.

Ready to submit your research? Choose BMC and benefit from:

- fast, convenient online submission

- thorough peer review by experienced researchers in your field

- rapid publication on acceptance

- support for research data, including large and complex data types

- gold Open Access which fosters wider collaboration and increased citations

- maximum visibility for your research: over $100 \mathrm{M}$ website views per year

At $\mathrm{BMC}$, research is always in progress.

Learn more biomedcentral.com/submission 